\title{
Immobilized glucose oxidase on boronic acid-functionalized hierarchically porous MOF as an integrated nanozyme for one-step glucose detection
}

\author{
Zhenghong Zhao, Yaojing Huang, Wenren Liu, Fanggui Ye* and Shulin Zhao \\ State Key Laboratory for the Chemistry and Molecular Engineering of Medicinal Resources, \\ College of Chemistry and Pharmaceutical Science of Guangxi Normal University, Guilin 541004, \\ P. R. China.
}

Total number of pages: 16

Total number of Figures: 10

Total number of Figures: 3 


\section{Instrument and chemicals}

Nearly all chemicals were of analytical grade and were used without further purification. 2-aminoterephthalic acid, $\mathrm{FeCl}_{3} \cdot 6 \mathrm{H}_{2} \mathrm{O}$ and lauric acid was purchased from Aladdin (Shanghai, China). 4-formylphenyboronic acid, TMB, anhydrous sodium acetate, dimethyl formamide (DMF) were purchased from Shanghai Chemical Reagent Company (Shanghai, China). Glucose and glucose oxidase (100 $\left.\mathrm{U} \mathrm{mg}^{-1}\right)$ were purchased from Shanghai Sangon Bioengineering Technology Service Co., Ltd. (Shanghai, China). Deionized (DI) water was produced by Millipore purification system (Bedford, MA, USA).

\section{Characterization}

Scanning electron microscopy (SEM) was conducted with a Quanta 200 FEG SEM (Philips, Netherlands). Transmission electron microscopy (TEM) was carried out on a Tecnai F-20 electron microscope operated at $200 \mathrm{kV}$ (FEI, USA). X-ray diffraction (XRD) patterns were obtained with an X' Pert PRO diffractometer (PANalytical, Netherlands) using CuKa radiation. X-ray photoelectron spectroscopy (XPS) data were obtained using a Thermo ESCALAB 250XI electron spectrometer (Thermo, USA) using 150-W AlK alpha radiation. Fourier transform infrared (FT-IR) spectra $\left(4000-400 \mathrm{~cm}^{-1}\right)$ in $\mathrm{KBr}$ were recorded using a PE Spectrum One FT-IR spectrometer (PE, USA). Thermogravimetric analysis (TGA) was conducted using a LABSYS evo TG-DSC/DTA instrument (Setaram Instrumentation, France). UV absorption spectra were recorded on a model Cary 60 spectrophotometer (Agilent, USA). 

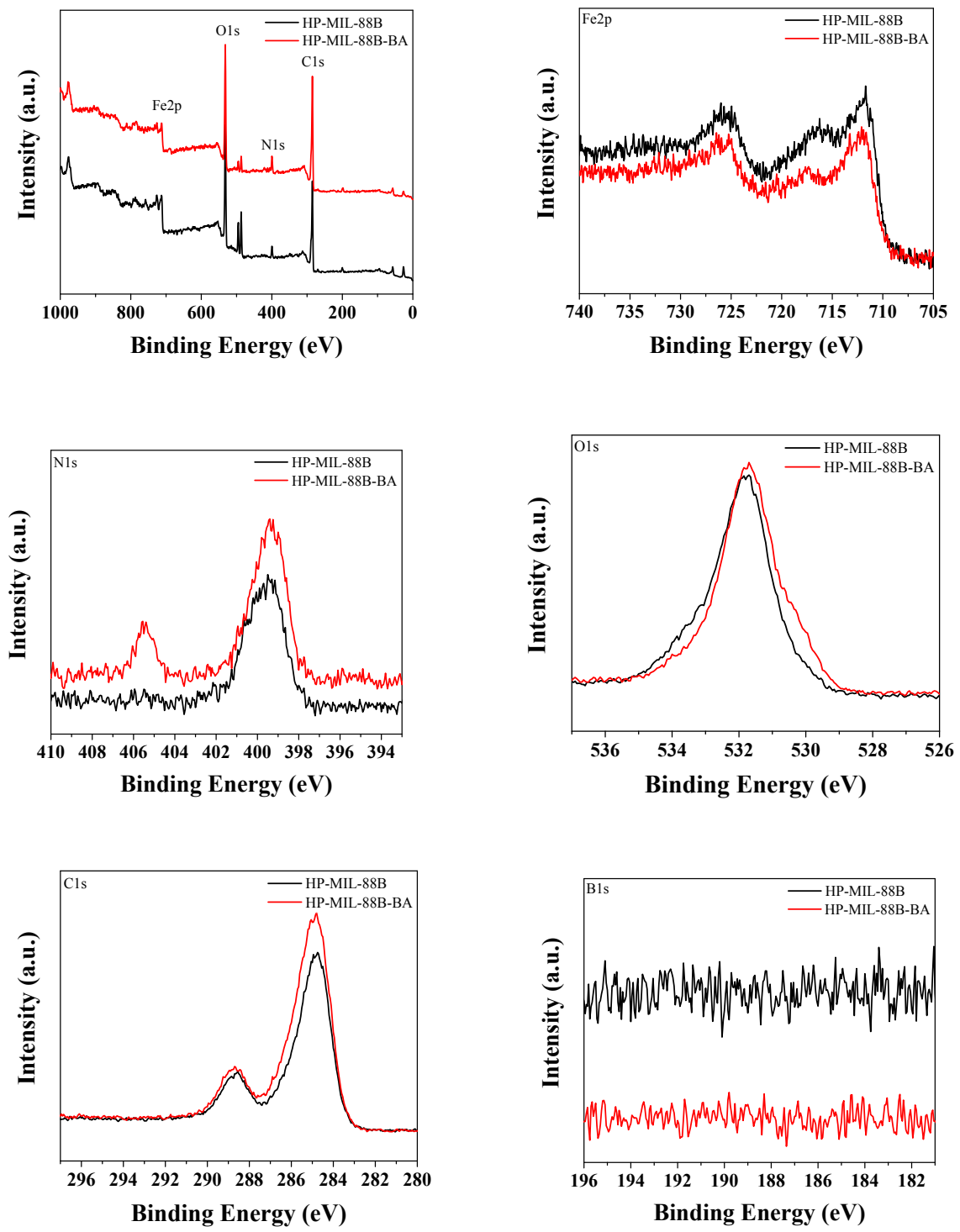

Figure S1. Wide-scan and regions XPS spectra for HP-MIL-88B and HP-MIL-88B-BA. 

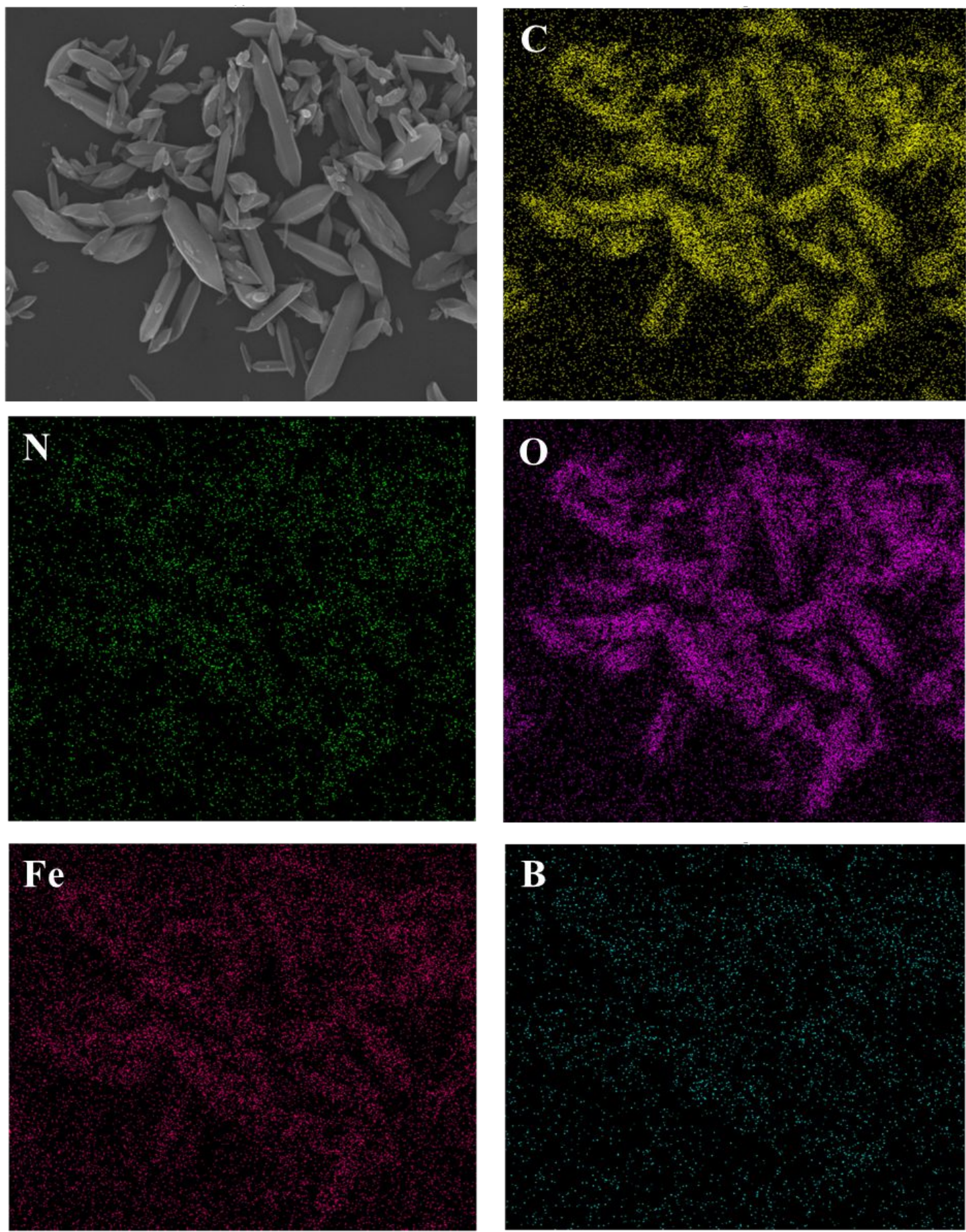

Figure S2. High-angle annular dark-field scanning transmission electron microscopy image image and elemental mapping for C, N, O, Fe and B of HP-MIL-88B-BA 


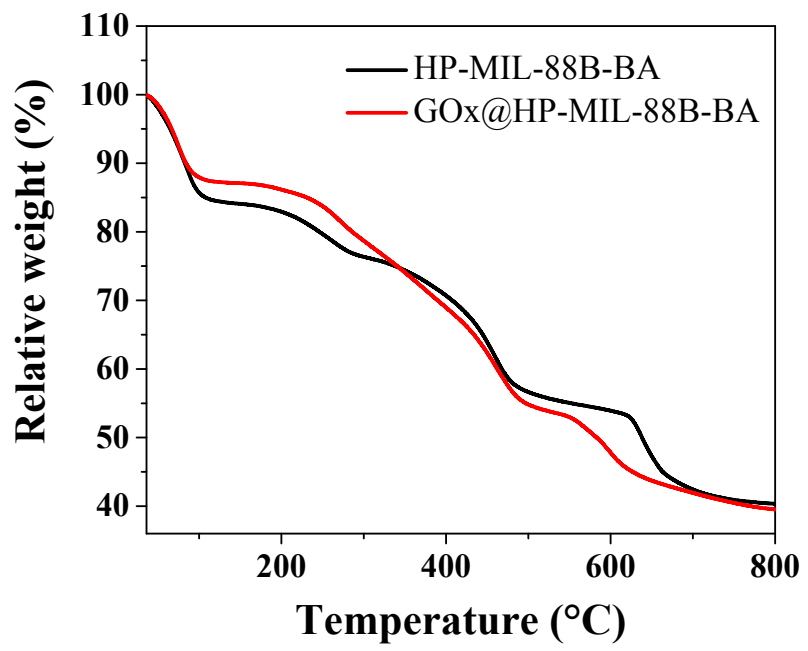

Figure S3. TGA curve of HP-MIL-88B-BA (black) and GOx@HP-MIL-88B-BA (red). 


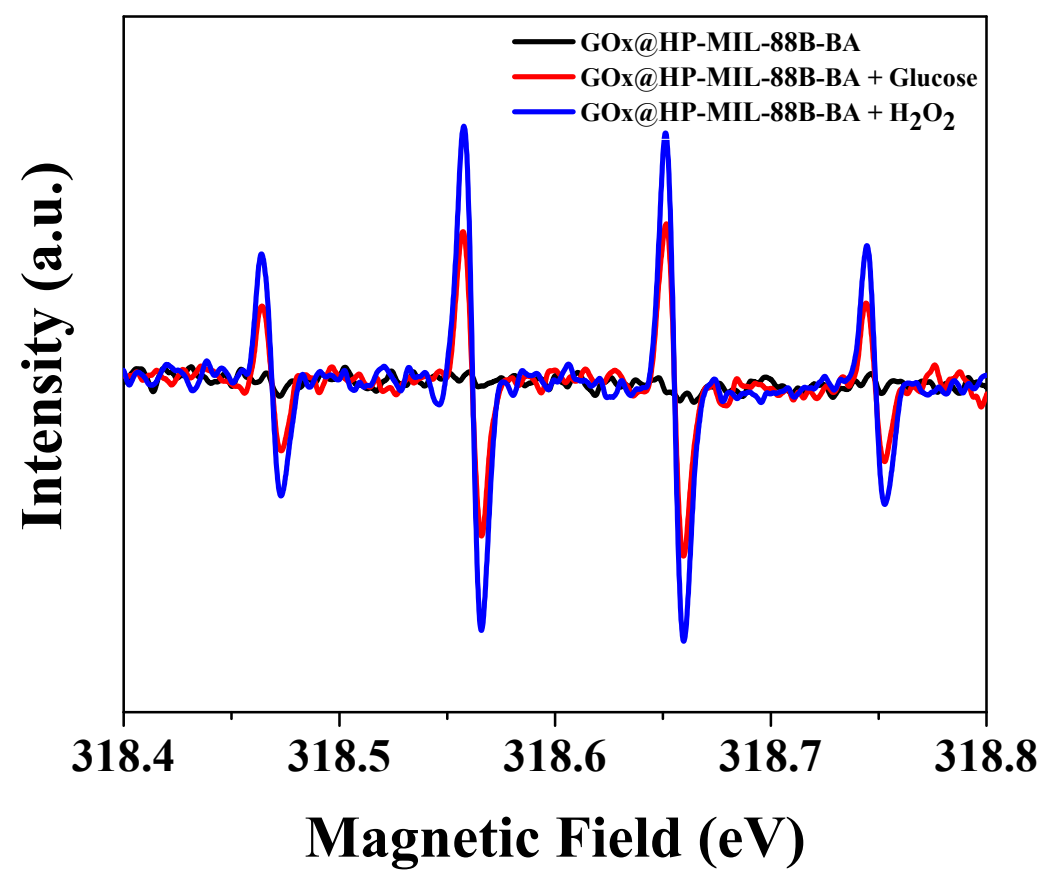

Figure S4. ESR spectra of $\bullet \mathrm{OH}$ radicals in different reaction systems with DMPO as the spin trap. 


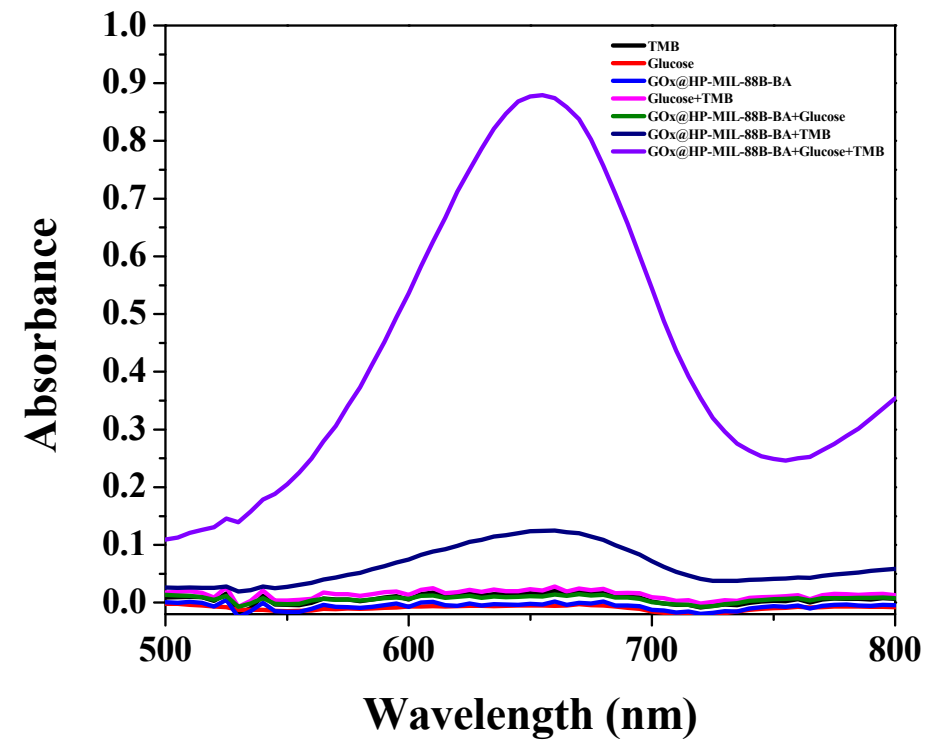

Figure S5. UV-visible absorption spectra of seven sample solutions in $0.010 \mathrm{~mol} \mathrm{~L}^{-1}$ acetate buffer (pH 4.0) containing TMB, Glucose, GOx@HP-MIL-88B-BA, Glucose + TMB, Glucose + GOx@HP-MIL-88B-BA, TMB + GOx@HP-MIL-88B-BA, Glucose + TMB + GOx@HP-MIL-88B-BA. 


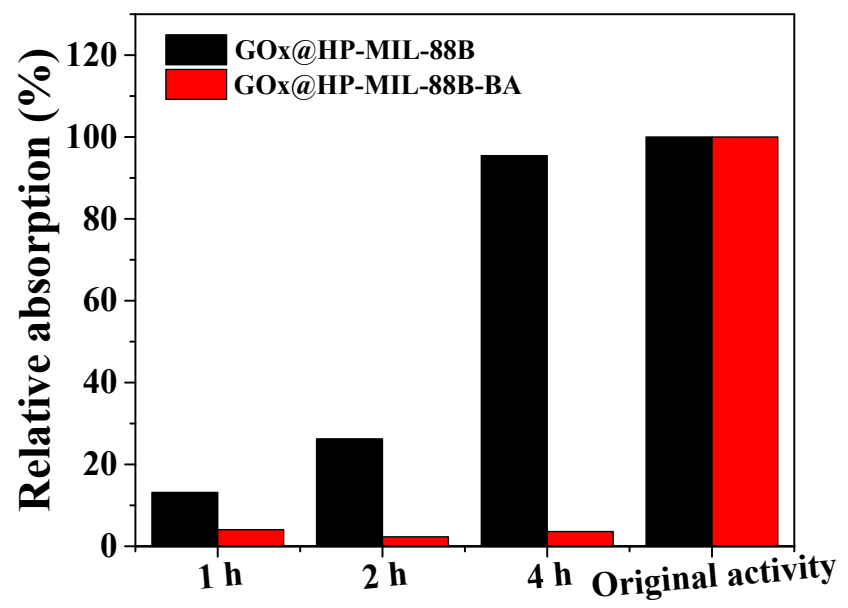

Figure S6. Enzyme leaching experiment: GOx@HP-MIL-88B and GOx@HP-MIL-88B-BA was immersed in acetic acid buffer for 1,2, $4 \mathrm{~h}$ and the supernatant was taken for enzyme activity test 
(a)

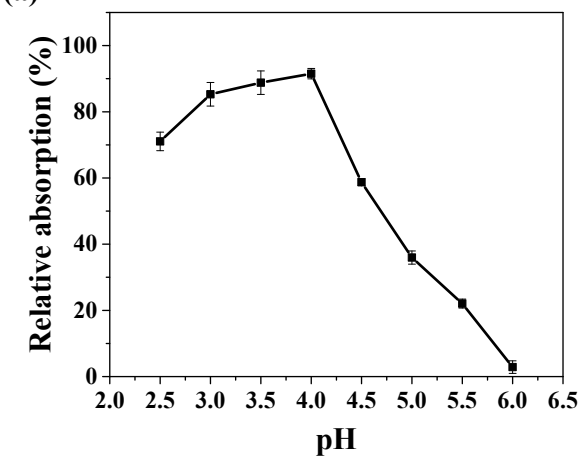

(c)

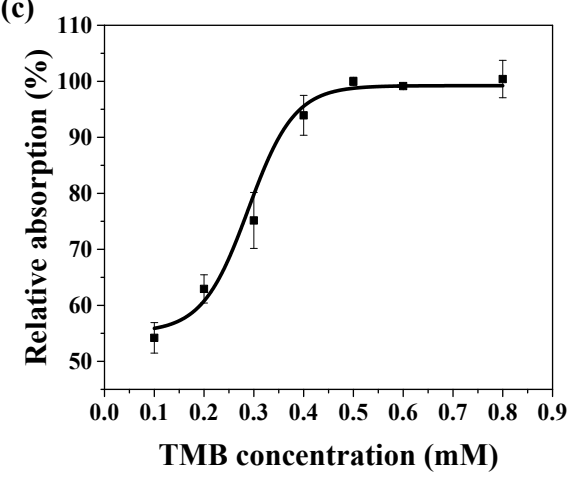

(b)

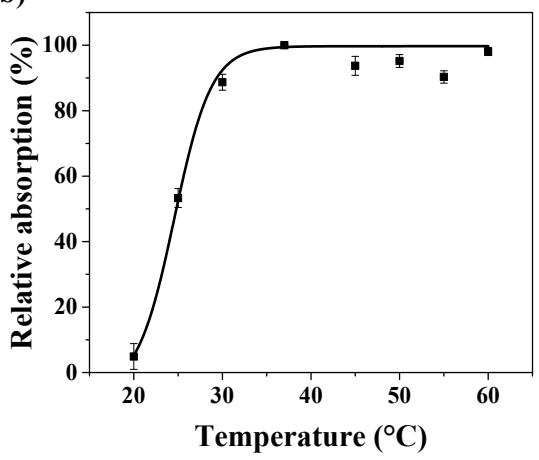

(d)

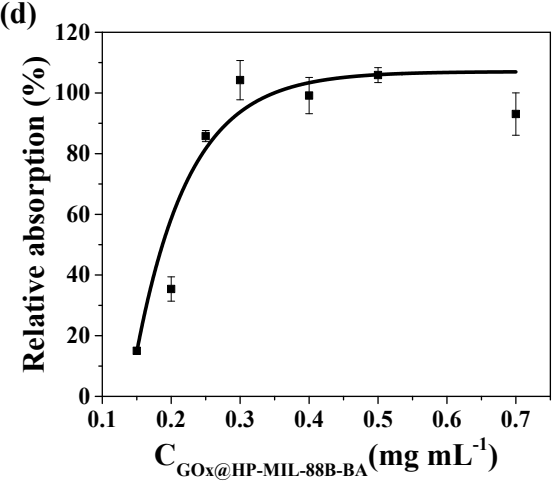

Figure S7. Effect of pH, reaction temperature, TMB concentrations and GOx@HP-MIL-88B-BA concentrations on the GOx@HP-MIL-88B-BA system for the glucose detection. The error bars represent the standard deviation of three measurements. 
(a)

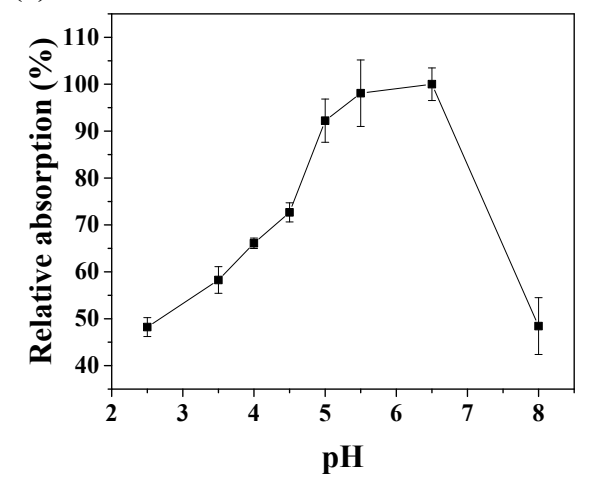

(b)

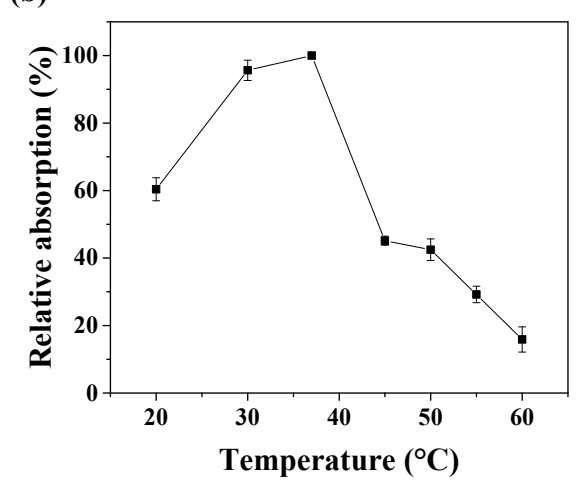

Figure S8. Effect of pH, reaction temperature on the free enzyme system for the glucose detection. The error bars represent the standard deviation of three measurements. 

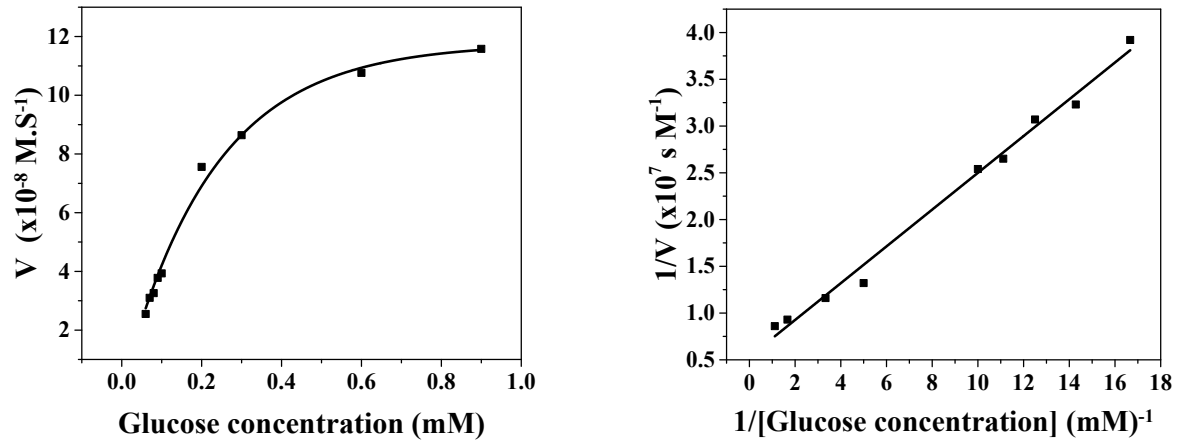

Figure S9. Steady-state kinetic analysis of GOx@HP-MIL-88B-BA. 

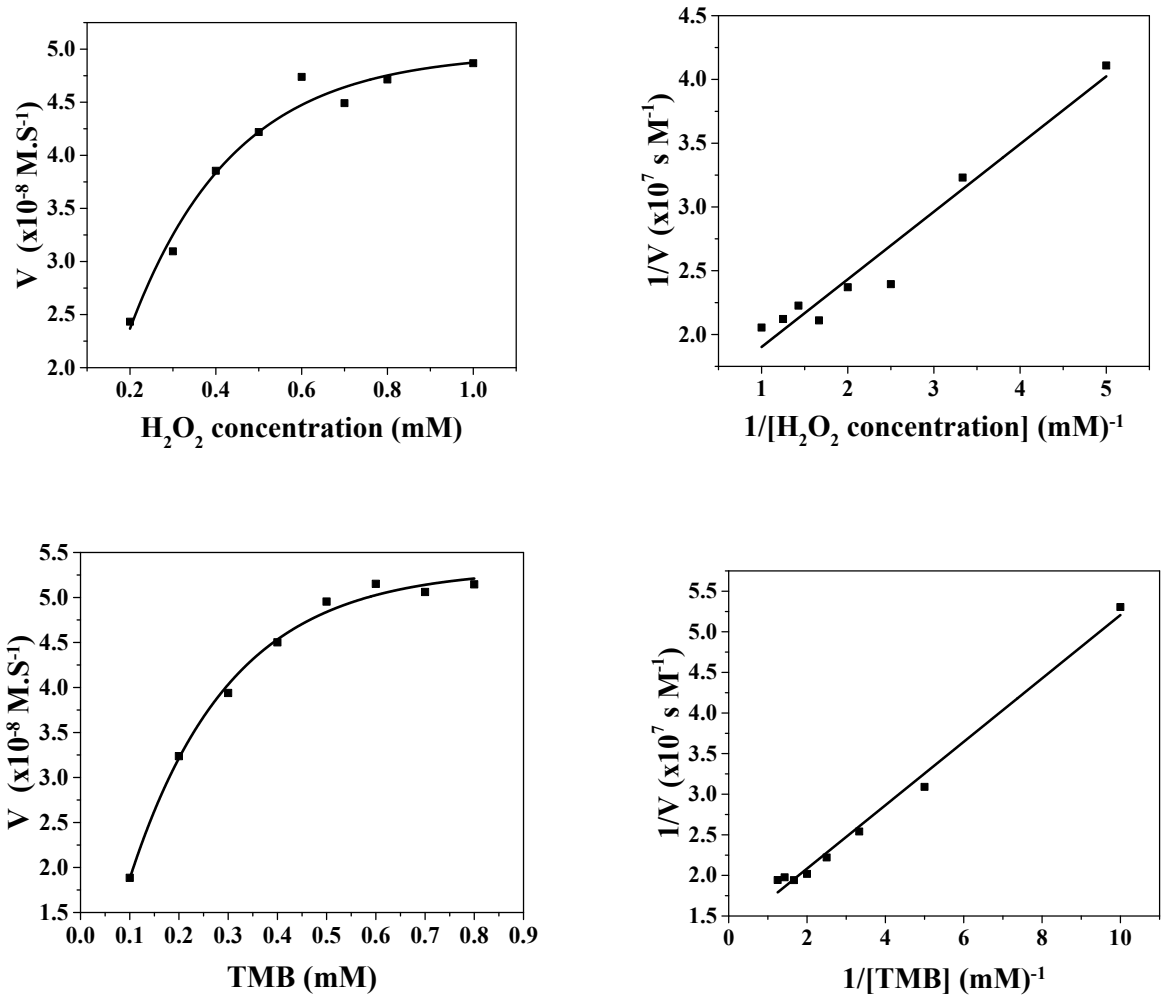

Figure S10. Steady-state kinetic analysis of the GOx@HP-MIL-88B-BA. 
Table S1 The amount of B of HP-MIL-88B-BA determined by ICP-MS

\begin{tabular}{ll}
\hline Sample & $\mathrm{B}$ \\
\hline HP-MIL-88B(Fe) (mg/kg) & 0.00 \\
HP-MIL-88B(Fe)-BA 20wt\% (mg/kg) & 122.42 \\
HP-MIL-88B(Fe)-BA 40wt\% (mg/kg) & 220.02 \\
HP-MIL-88B(Fe)-BA 100wt\% (mg/kg) & 323.59 \\
\hline
\end{tabular}


Table S2 Comparison the analytical performance of the GOx@HP-MIL-88B-BA with that of other reported integrated nanozymes for glucose detection

\begin{tabular}{|c|c|c|c|c|c|}
\hline Catalyst & $\begin{array}{c}\text { Linear } \\
\text { range }(\mu M)\end{array}$ & $\begin{array}{l}\text { Detection } \\
\operatorname{limit}(\mu M)\end{array}$ & $\begin{array}{c}\text { Catalyst } \\
\text { concentration }(\mu \mathrm{g} \\
\left.\mathrm{mL}^{-1}\right)\end{array}$ & $\begin{array}{c}\text { Reaction } \\
\text { time (min) }\end{array}$ & Ref \\
\hline Fe-MOF-GOx & $1-500$ & 0.487 & 33 & 30 & [1] \\
\hline GOx@ZIF-8(NiPd) & $10-300$ & 9.2 & 1000 & 60 & [2] \\
\hline $\begin{array}{c}\text { GOx@ZIF-8@Fe-PD } \\
\text { A }\end{array}$ & $5-100$ & 1.1 & 390 & 60 & [3] \\
\hline GOx@Fe (III)-BTC & $5-100$ & 2.4 & 240 & 60 & [4] \\
\hline GOx/hemin@ZIF-8 & $0-240$ & 1.7 & 200 & 60 & [5] \\
\hline $\begin{array}{c}\text { GOx@HP-MIL-88B- } \\
\text { BA }\end{array}$ & $2-100$ & 0.98 & 300 & 10 & In this work \\
\hline
\end{tabular}

[1] Xu, W.; Jiao, L.; Yan, H.; Wu, Y.; Chen, L.; Gu, W.; Du, D.; Lin, Y.; Zhu, C. Glucose oxidase-integrated metal-organic framework hybrids as biomimetic cascade nanozymes for ultrasensitive glucose biosensing. ACS Appl. Mater. Interfaces 2019, 11, 22096-22101. DOI: 10.1021/acsami.9b03004

[2] Wang, Q.; Zhang, X.; Huang, L.; Zhang, Z. GOx@ZIF-8 (NiPd) nanoflower: an artificial enzyme system for tandem catalysis. Angew. Chem. Int. Ed. 2017, 129, 16298-16301. DOI: 10.1002/anie. 201710418

[3] Zhao, Z.; Lin, T.; Liu, W.; Hou, L.; Ye, F. and Zhao, S. Colorimetric detection of blood glucose based on GOx@ZIF-8@Fe-polydopamine cascade reaction. Spectrochim.Acta.A Mol. Biomol. Spectrosc. 2019, 219, 240-247. DOI: 10.1016/j.saa.2019.04.061

[4] Zhao, Z.; Pang, J.; Liu, W.; Lin, T.; Ye, F.; Zhao, S. A bifunctional metal organic framework of type Fe (III)-BTC for cascade (enzymatic and enzyme-mimicking) colorimetric determination of glucose. Microchim. Acta 2019, 186, 295. DOI: 10.1007/s00604-019-3416-7

[5]Cheng, H.; Zhang, L.; He, J.; Guo, W.; Zhou, Z.; Zhang, X.; Nie, S.; Wei, H. Integrated nanozymes with nanoscale proximity for in vivo neurochemical monitoring in living brains. Anal. Chem. 2016, 88, 5489-5497. DOI: 10.1021/acs.analchem.6b00975 
Table S3 Maximum reaction rate $\left(\mathrm{V}_{\max }\right)$ and Michaelis constant $\left(\mathrm{K}_{\mathrm{m}}\right)$ data of GOx and GOx@HP-MIL-88B-BA.

\begin{tabular}{|c|c|c|c|c|}
\hline Catalyst & Substrate & $\mathbf{K}_{\mathbf{m}} / \mathbf{m m o l ~ L}^{-1}$ & $V_{\max } / 10^{-7} \mathrm{~mol} \mathrm{~L}^{-1} \mathrm{~s}^{-1}$ & Ref \\
\hline GOx & Glucose & 5.2 & 1.89 & \\
\hline \multirow[t]{2}{*}{ HRP } & $\mathrm{H}_{2} \mathrm{O}_{2}$ & 1.6 & 2.89 & {$[1]$} \\
\hline & TMB & 0.86 & 2.31 & \\
\hline \multirow[t]{3}{*}{ GOx@HP-MIL-88B-BA } & Glucose & 0.37 & 2.0 & \\
\hline & $\mathrm{H}_{2} \mathrm{O}_{2}$ & 0.38 & 0.72 & In this work \\
\hline & TMB & 0.22 & 0.68 & \\
\hline
\end{tabular}

[1] Zhao, Z.; Pang, J.; Liu, W.; Lin, T.; Ye, F.; Zhao, S. A bifunctional metal organic framework of type Fe (III)-BTC for cascade (enzymatic and enzyme-mimicking) colorimetric determination of glucose. Microchim. Acta 2019, 186, 295. DOI: 10.1007/s00604-019-3416-7 
Table S4 The recovery test of glucose in diluted serum samples

\begin{tabular}{|c|c|c|c|c|c|}
\hline Sample & $\begin{array}{c}\text { Conventional } \\
\text { enzymatic method }^{\mathrm{b}} \\
(\mathbf{m M})\end{array}$ & $\begin{array}{c}\text { Added } \\
(\mathrm{mM})\end{array}$ & $\begin{array}{l}\text { Proposed method } \\
(\mathrm{mM}) \mathrm{Mean}^{\mathrm{c}} \pm \mathrm{SD}^{\mathrm{d}}\end{array}$ & $\begin{array}{c}\text { Recovery } \\
\text { (\%) }\end{array}$ & $\operatorname{RSD}(\%)$ \\
\hline \multirow{3}{*}{ Sample1 } & \multirow{3}{*}{6.64} & 0.8 & $7.48 \pm 0.03$ & 105.0 & 3.8 \\
\hline & & 1.6 & $8.34 \pm 0.13$ & 106.2 & 1.6 \\
\hline & & 2.4 & $9.10 \pm 0.11$ & 102.5 & 1.2 \\
\hline \multirow{3}{*}{ Sample2 } & \multirow{3}{*}{5.07} & 0.8 & $5.79 \pm 0.10$ & 90.0 & 1.7 \\
\hline & & 1.6 & $6.58 \pm 0.24$ & 94.4 & 3.6 \\
\hline & & 2.4 & $7.48 \pm 0.13$ & 100.4 & 1.7 \\
\hline \multirow{3}{*}{ Sample3 } & \multirow{3}{*}{7.30} & 0.8 & $8.15 \pm 0.24$ & 106.3 & 2.9 \\
\hline & & 1.6 & $8.99 \pm 0.18$ & 105.6 & 2.0 \\
\hline & & 2.4 & $9.83 \pm 0.28$ & 105.4 & 2.8 \\
\hline
\end{tabular}

a The serum samples were obtained from the Guilin Hospital of Chinese Traditional and Western Medicine.

${ }^{\mathrm{b}}$ The glucose determination was performed by the proposed method.

${ }^{\mathrm{c}} \mathrm{n}=3$

d SD: Standard Deviation 